\title{
Estudios multidisciplinarios: un esbozo de la investigación cultural en la Universidad Tecnológica de El Salvador, 1999-2013
}

\author{
Multidisciplinary studies: an outline of cultural research at the \\ Universidad Tecnológica de El Salvador, 1999-2013
}

\author{
"Una verdadera universidad requiere [...] el ejercicio de la investigación." \\ (Hernández, 2003, p. 192). \\ José Heriberto Erquicia-Cruz' \\ Director del Museo Nacional de Antropología "David J. Guzmán” \\ jose.erquicia@utec.edu.sv \\ Recibido: 06/03/2015 - Aceptado: 16/03/2015
}

\section{Resumen}

Las instituciones de educación superior están obligadas a desarrollar investigaciones en las diversas áreas del conocimiento que imparten. Es por ello que, desde diversas instancias de la Universidad Tecnológica de EI Salvador, se ha llevado a cabo una variedad de estudios en temas de cultura. El enfoque multidisciplinario en el abordaje de las investigaciones ha brindado un conocimiento mucho más amplio, crítico y colegiado de los problemas que se deben resolver. Estudios de identidades étnicas y urbanas, religiosidad popular, medicina tradicional, cultura material, biográficos, históricos literarios, históricos-económicos, etnográficos, patrimonio cultural, fuentes históricas y migrantes son algunos de los tópicos que se han logrado desarrollar en la última década y media de investigación cultural en la universidad.

\section{Palabras clave}

Estudios multidisciplinarios, investigación cultural, Universidad Tecnológica de El Salvador, educación superior.

\section{Abstract}

Institutions of higher education are required to develop research in the different fields of study they teach. Therefore, Universidad Tecnológica de El Salvador has conducted a variety of studies on issues of culture with the assistance of its different departments. The multidisciplinary approach in addressing research has provided a much broader, critical and professional knowledge of the problems that need to be solved. Some of the topics that the university has been able to develop in the last decade and a half of cultural research include the following: studies on ethnic and urban identities, popular religiosity, traditional medicine, material culture; biographical, historical, literary, historical-economic, and ethnographic studies, research in cultural heritage and historical and migrant sources.

\section{Keywords}

Multidisciplinary studies, cultural Research, Universidad Tecnológica de El Salvador, higher education.

José Heriberto Erquicia-Cruz, arqueólogo. Actualmente es el Director del Museo Nacional de Antropología "David J. Guzmán", también se desempeña como docente e investigador en la Universidad Tecnológica de El Salvador. Correo electrónico: jose.erquicia@utec.edu.sv 


\section{Introducción}

El presente ensayo aborda la producción investigativa de estudios de cultura desde diversas perspectivas de las Ciencias Sociales y las Humanidades, que se han desarrollado entre 1999 y 2013 en la Universidad Tecnológica de El Salvador, y desde varias de sus instancias.

En El Salvador, en seguida de los Acuerdos de Paz firmados en 1992, se conjeturaba una nueva etapa de desarrollo de la sociedad, este momento vino a hacer un "hiato" en la historia moderna del país. Desde el Estado se creaban nuevas políticas encaminadas a fortalecer y mejorar la calidad de vida de sus ciudadanos. Aspectos económicos, sociales, políticos y culturales, entre otros, venían a fortalecer la incipiente democracia, que se estaba construyendo. Uno de los principales aspectos para el desarrollo de la población de las naciones modernas son las apuestas por la educación. Es en este sentido que, en 1995, se realiza una nueva reforma educativa por parte del Ministerio de Educación (Mined). Dicha reforma también incluiría la Ley de Educación Superior (LES, 1995), la que es aprobada a finales del mismo año.

Dentro de este marco de la Ley de Educación Superior, el artículo 3 propone las funciones que debe tener la docencia universitaria en El Salvador; así, todas las universidades funcionarán bajo la tríada de: Docencia, Investigación científica y Proyección social. Para esta ley, la investigación "es la búsqueda sistemática y análisis de nuevos conocimientos para enriquecer la realidad científica y social" (LES, 1995).

De esta manera, las universidades salvadoreñas, conforme a la LES, han de contar con docentes que tengan competencias y experiencias en la investigación (Artículo 38. LES, 1995), ya que de esa manera podrán transmitirla a sus estudiantes; y que dicho conocimiento sea una herramienta más de la formación profesional con la que todo estudiante universitario debe contar.

Algunos de los nuevos enfoques de la educación a nivel superior hablan de la gran importancia de la investigación como "el único camino para incrementar el saber [en la educación] es la investigación" (Parra, 2004, p.65). En este sentido, se logra certificar que no puede pensarse una universidad sin investigación, pues de ahí germina el saber. Desde hace casi dos décadas, en el proceso de enseñanzaaprendizaje, ha sido fundamental, para la formación de profesionales, tomar la investigación como eje transversal para el desarrollo de aprendizajes significativos en los estudiantes.

En el ámbito internacional, durante la Conferencia Mundial de Educación Superior, desarrollada en París en julio de 2009, se ratificó "la importancia de la educación superior y la investigación para hacer frente a los desafíos mundiales y construir economías basadas en el conocimiento que sean más integradoras, equitativas y sostenibles" (CMES, 2009).

Por su parte, la Utec ha impulsado dos formas de investigaciones: la institucional y la de cátedra. La de cátedra, o también conocida como formativa, es investigación generada a partir de los contenidos temáticos; se planifican dentro del diseño instruccional de las asignaturas. Ello implica el diseño de un proceso de investigación planificada, bajo la responsabilidad y dirección del docente, la cual es ejecutada por los estudiantes de la asignatura correspondiente (Erquicia, 2011, p.7).

En este ensayo se presentarán investigaciones que surgen de las dos modalidades, investigaciones institucionales y de cátedra, que en su momento han sido publicadas en diversos espacios de difusión de la Utec.

Los inicios de los estudios históricos y antropológicos desde la investigación institucional (19992003)

Una vez conformada una dirección de investigaciones, como ente que regiría la investigación dentro de la universidad, inician los primeros estudios históricos y antropológicos desde el centro de investigación. El estudio denominado "La gesta de Anastasio Aquino: una aproximación histórica" (Barraza Ibarra, 2001) es un documento que describe el entorno geográfico de la región conocida como Los Nonualcos, en la zona paracentral de El Salvador; luego hace un retrato histórico-literario del personaje Anastasio Aquino, así como reseña los antecedentes y la rebelión misma hasta la muerte de Aquino.

Por su parte, el trabajo dividido en dos segmentos, "Breve esbozo histórico de los indígenas en El Salvador" (I y II) (Ramos, 2000, 2001) emprende la temática de la negación del indígena en El Salvador, así como los aspectos de territorio y población; las representaciones religiosas; la visión del mundo; los primeros años de la presencia española 
Estudios multidisciplinarios: un esbozo de la investigación cultural en la Universidad Tecnológica de El Salvador, 1999-2013. José Heriberto Erquicia-Cruz, pp. 8-15.
Revista entorno, abril 2015, número 58.

ISSN: 2218-3345. en actual territorio salvadoreño; luego los indígenas en la producción de cacao; para terminar con el papel de los indígenas en el proceso de independencia. La segunda parte retoma la situación de los indígenas en los primeros años de la independencia (1821-1838). Para luego adentrarse en la era republicana y la introducción del café (1840-1932), terminando con la desesperación obligada al resurgimiento indígena de 1932 hasta el final del siglo XX.

Quizá uno de los proyectos más ambiciosos que se inició en este período, y que finalizó hasta la publicación completa en 2006, fue la serie de cinco tomos de Historia de la economía de la provincia del Salvador desde el siglo XVI hasta nuestros días (Barraza Ibarra, 2006). Esta serie de tomos, como su título lo dice, emprende una descripción de las economías de la provincia de San Salvador, el Estado del Salvador, hasta El Salvador del siglo XX.

Desde el abordaje antropológico se publicó el artículo "La sobrevivencia de la medicina tradicional como alternativa en los sectores populares. Magia, hechicería y medicina tradicional" (Ramos, 2004), que surge de la necesidad de investigar cuáles son las vías alternas para mejorar el estado de salud del pueblo salvadoreño. Con ello, la autora afirma que

en el preámbulo histórico, [la] evolución de la medicina tradicional en el país, desembocando en la conclusión de que medicina tradicional salvadoreña, es una medicina sincrética, ya que reúne la herencia milenaria de los pueblos indígenas que poblaron nuestro territorio, la herencia europea y árabe aportada por los españoles y una pequeña contribución de los africanos traídos en calidad de esclavos (Ramos, 2004, p. 31).

La investigación concluye que la medicina tradicional constituye una alternativa factible de curación para los sectores populares en combinación con la medicina oficial.

\section{La Escuela de Arte y Cultura y las investigaciones de cátedra (2004-2006)}

A inicios del nuevo siglo, la Utec emprendía el proyecto de creación de las denominadas "carreras de la cultura", las cuales verían luz a través de la Escuela de Arte y Cultura, la que a partir de 2006 llevaría el nombre de "Escuela de
Antropología". La demanda de formar profesionales en Antropología, Arqueología e Historia se hacía más urgente en la época en que el país estaba atravesando una de las etapas más dinámicas de su historia, cuyos cambios políticos, sociales, económicos y culturales requerían con urgencia la labor de estos profesionales, especialmente para impulsar el desarrollo de la investigación sobre las diversas culturas: sus valores, sus costumbres, sus modos de vivir $y$, en definitiva, sus identidades culturales.

El impulso a la investigación, y en específico a las investigaciones de cátedra, desde la llegada del antropólogo Ramón Rivas a la dirección de la Escuela de Arte y Cultura, dio un giro importante hacia la publicación de varios estudios que se divulgaron entre 2004 y 2006, con la denominada colección de estudios antropológicos, arqueológicos e históricos.

De la colección antropológica se desprenden las siguientes investigaciones. "Maras y transculturación. Orígenes de la violencia entre las pandillas juveniles en Ilobasco, El Salvador" (Rivas, 2004). Este texto analiza el caso de las maras como un fenómeno de grupos sociales, que por sus características se ubican al margen del contexto político, social, cultural y económico de El Salvador. Tomando como punto de arranque que el origen de las pandillas juveniles radica en los procesos de exclusión y marginación en los que ha vivido la juventud salvadoreña por parte del Estado, íntimamente vinculada con la socialización e interiorización de la violencia familiar.

Por su parte, en "Los retos de la antropología en Centroamérica" (González, 2004) se esboza una muestra de la antropología como compromiso social, una antropología inquieta, que cuestiona, la cual no se percibe como un fin, sino como un medio para la transformación de la sociedad, y que a su vez es propositiva incidiendo en la responsabilidad de la disciplina con los derechos humanos y especialmente con los desfavorecidos de la región centroamericana.

"Deseo local y patrimonio histórico en Suchitoto" (Rivas, et al, 2005), fue producto de una investigación de cátedra de la asignatura Antropología Social. Este trabajo consiste en una investigación etnográfica de municipio de Suchitoto, del departamento de Cuscatlán, en el cual se aborda su potencial turístico, la organización socioeconómica, la historia local y el patrimonio cultural. Asimismo, otros elemento importante 
Estudios multidisciplinarios: un esbozo de la investigación cultural en la Universidad Tecnológica de El Salvador, 1999-2013. José Heriberto Erquicia-Cruz, pp. 8-15.
Revista entorno, abril 2015, número 58.

ISSN: 2218-3345. es el relativo a la memoria histórica y a las relaciones con los otros municipios. Otra de las investigaciones de cátedra de la asignatura de Expresiones Folclóricas es "Breve estudio del proceso de elaboración y distribución de la alfarería utilitaria de Quezaltepeque" (De Gutiérrez, et al, 2005). Esta monografía de Quezaltepeque muestra un bosquejo general de los aspectos económicos, sociales, culturales, políticos e históricos del municipio, en donde, además, se recogieron los testimonios de los artesanos trabajadores y dueños de los talleres de alfarería; con ello pretendían analizar y construir una nueva visión sobre el estado de la producción de alfarería utilitaria, y así poder aportar a la reconstrucción de la historia sociocultural de la zona.

Entre las temáticas de las identidades, en "La ausencia del otro. Las personas indígenas en la legislación salvadoreña" (Cañas-Dinarte, 2006) se abordan particularmente los proyectos de las legislaciones desde la segunda mitad del siglo $\mathrm{XX}$, que sin embargo para las comunidades indígenas de El Salvador no ha representado una oportunidad de inclusión a los proyectos de nación. Pues aunque los pueblos indígenas salvadoreños cuenten con el reconocimiento de parte del Estado, carecen de formas de divulgación de sus pensamientos, sentimientos y quehaceres cotidianos. La segunda parte, "Conceptualización e interpretación de la 'definición' indígena en El Salvador" (Rivas, 2006), realiza un recorrido teórico por las diversas definiciones y conceptos de indígena que a través de diversas fuentes se han descrito en El Salvador.

"Antropología y arqueología de la isla Conchagüita en el golfo de Fonseca" (Rivas y Gómez, 2005) presenta un estudio de la isla de Conchagüita desde una perspectiva antropológica de las comunidades que residen en la actualidad en la isla, para luego prestar atención al estudio de los materiales culturales del sitio arqueológico colonial de Conchagua Vieja, antigua iglesia de Santiago de Conchagua.

La Colección Arqueológica inició con el título "Identidad española reflejada en una casa colonial de la antigua villa de San Salvador (1528-1545)" (Gallardo, 2005). En este estudio se emprende un recorrido por la historia de la antigua villa de San Salvador del valle de La Bermuda, ahondando en la identidad española a partir de los restos materiales $y$ de las relaciones de poder que se ejercían para imponer su cultura. El otro texto de arqueología es "Investigaciones arqueológicas en la zona del golfo de Fonseca, El Salvador" (Erquicia, 2005). Dicho estudio es una recopilación de datos que brinda un panorama introductorio y general que comprende el ámbito geográfico y cultural del sector salvadoreño del golfo de Fonseca, enfocado especialmente en la investigación arqueológica desarrollada en los últimos años en la zona en mención.

Por último, pero no por eso menos importante, la Colección Histórica inicia con el tema "Los pipiles en Metapán y su zona de influencia a través de las fuentes coloniales" (López-Lago y Benítez, 2005). Este texto lleva al lector por un recorrido desde las fuentes del siglo XVI hasta las de la primera mitad del siglo XIX, las cuales incluyen la Relación Marroquín, las Tasaciones de Cerrato, el Memorial de los encomenderos, la carta de Diego García de Palacio dirigida a Felipe II, la Relación Breve de Fray Alonso Ponce, la Recordación Florida de Fuentes y Guzmán, la Relación de Manuel Gálvez del Corral, alcalde mayor de San Salvador, la Descripción geográfico-moral de Cortés y Larraz, el Estado de la provincia de San Salvador del intendente Antonio y Gutiérrez, finalizando con el Compendio de historia de Domingo Juarros. Otro de los estudios históricos es Estado, economía y redes de poder en el oriente salvadoreño, 18761915 (Campos Lara, 2006). Este trabajo se enmarca en los estudios que comprenden la construcción e integración del Estado-nación salvadoreño, particularmente en la región oriental de El Salvador, entre 1876 y 1915, y aborda cómo el poder económico se encontraba en la tenencia de la tierra, la agricultura, la ganadería y el comercio, los cuales estaban en las manos de familias que ejercieron una influencia local y regional.

\section{La Colección Ciencias Sociales (2008-2013)}

Esta colección brinda un panorama de investigaciones surgidas desde la Escuela de Antropología de la Facultad de Ciencias Sociales y desde el Museo Universitario de Antropología de la Utec. Así, "Etnografía de salvadoreños migrantes en Brentwood y Hempstead, Nueva York" (Colorado, 2008) ofrece una aproximación etnográfica de las comunidades de migrantes salvadoreñas ubicadas en Hempstead y Brentwood en Long Island, estado de Nueva York, Estados Unidos de América durante 2005. El estudio se fundamentó en observaciones participantes y entrevistas abiertas que ofrecieron las miradas y expresiones de hechos personales, íntimos e históricos de los entrevistados, como migrantes. Por su parte, "Medicina tradicional entre los indígenas de Izalco, Sonsonate, El Salvador" (Castillo, 2012) es un trabajo etnográfico que pone en la palestra el legado 
Estudios multidisciplinarios: un esbozo de la investigación cultural en la Universidad Tecnológica de El Salvador, 1999-2013. José Heriberto Erquicia-Cruz, pp. 8-15.
Revista entorno, abril 2015, número 58.

ISSN: 2218-3345. cultural de los pueblos indígenas que aún subsisten en El Salvador. Se trata de la medicina tradicional, fundamental para el ser humano y que es parte de la cultura material e inmaterial de las comunidades. Este, a su vez, constituye un trabajo de recopilaciones de las especies vegetales, animales y minerales usadas con fines medicinales y terapéuticos entre los pobladores de Izalco. Cabe resaltar que los dos trabajos anteriores emergen de tesis de licenciatura en Antropología de cada uno de los autores.

Siempre de la Colección Ciencias Sociales, "Antropología en El Salvador. Recorrido histórico y descriptivo" (Rivas, 2012) exhibe una serie de ensayos que pretenden suscitar la comprensión de las Ciencias Sociales como ciencias que entregan una explicación a las diversas problemáticas de la sociedad salvadoreña, y como el mismo autor expresa: "Este es un ensayo sobre lo que podría denominarse recorrido histórico y descriptivo para la comprensión de los estudios de la gente y su entorno sociocultural" (Rivas, 2012, p. 23). El último trabajo de investigación publicado de esta serie se denomina llobasco de los recuerdos. De las muchas cosas que pasaban (Rivas, 2013), texto que consiste en una recopilación de historias locales desde los pobladores de llobasco y desde el mismo autor: los recuerdos, la tradición oral, la memoria colectiva, la oralitura y la oralidad; a partir de técnicas de investigación desde el tratamiento de las fuentes escritas y orales.

Continuidad de los estudios antropológicos, arqueológicos e históricos desde la investigación institucional (2006-2013)

A partir de 2006, desde la Dirección de Investigaciones de la Vicerrectoría de Investigación, hay un nuevo empuje en el desarrollo de la línea de investigación en historia, patrimonio cultural e identidades, con un enfoque multidisciplinario. La "Investigación arqueológica de restos arquitectónicos en Ciudad Vieja y diagnóstico de la valoración patrimonial del caserío El Molino y comunidad Primavera con el sitio histórico Ciudad Vieja, Suchitoto, Cuscatlán, El Salvador" (Erquicia et al, 2006) radicó en el desarrollo de un sondeo arqueológico en la calle de acceso al sitio, encontrándose cuatro estructuras de diversas funciones; al mismo tiempo se realizó el diagnóstico de la valoración patrimonial de los caseríos El Molino y la comunidad Primavera.

Desde la arqueología histórica se han desarrollado tres fases de las investigaciones denominadas "Proyecto de registro y reconocimiento de sitios arqueológicos históricos de El Salvador" (Erquicia, 2008, 2009 y 2012), las cuales han registrado e inventariado treinta y un sitios arqueológicos históricos, que van desde haciendas, iglesias, capillas, ingenios de hierro, beneficios de café, obrajes de añil, antiguos asentamientos coloniales y demás yacimientos arqueológicos históricos localizados dentro del territorio salvadoreño.

Por su parte, en la temática de la arqueología prehispánica, el trabajo "Proyecto migraciones nahua-pipiles del postclásico en la Cordillera del Bálsamo" (Escamilla y Fowler, 2013) formula que, aunque es difícil establecer una fecha exacta de la llegada de los pipiles a Centroamérica, existe evidencia lingüística, histórica y arqueológica que indica una fuerte migración pipil durante el postclásico temprano (900-1200 DC). Para el período de la Conquista (1524), los grupos nahua-pipil se encontraban localizados en el sureste de la costa Pacífica centroamericana, sureste de las tierras altas de Guatemala y específicamente en la parte central y oeste de El Salvador. En el estudio se analizan los recientes descubrimientos de sitios arqueológicos postclásicos registrados en la Cordillera del Bálsamo desde una perspectiva de la arqueología del paisaje. Así mismo, se discuten diferentes modelos interpretativos acerca del masivo movimiento migratorio nahua-pipil. $Y$ se riñe la probabilidad que la Cordillera del Bálsamo fue interpretada como el lugar idóneo para el desarrollo de apropiaciones del paisaje en términos defensivos y simbólicos, como parte de un proceso de emulación, con la finalidad de conservar prácticas culturales identitarias.

En la investigación de temas antropológicos e históricos, se ha desarrollado una serie de exploraciones. Así, en "Aproximación etnográfica al culto popular del hermano Macario en Izalco, Sonsonate, El Salvador" (Erquicia y Herrera, 2011), la investigación se sitúa en la comunidad multiétnica del municipio de Izalco. Dicho lugar cuenta con tradiciones religiosas y creencias populares que provienen desde hace centurias. Es así que durante el siglo XX surge el culto y creencias en torno al Hermano Macario, que poco a poco ha ido fortaleciéndose, contexto en el que sus creyentes y practicantes conviven en un ambiente con diversidad de credos. Este personaje, según la tradición oral, es un indígena curandero que aún después de muerto sigue sanando a quienes lo invocan. En la actualidad, los agradecimientos por los favores recibidos son expresados por medio de cantos, rituales y otras acciones orientadas al 
personaje que surgió de la misma población del municipio. Tan importante es el culto para esta comunidad de creyentes que han creado un día especial para conmemorarlo; visitan su tumba, que se ubica en el cementerio local, y en ella dejan diferentes ofrendas, que son llevadas durante todo el año. Esta investigación es una aproximación al estudio de las creencias populares particulares de la comunidad de Izalco.

En 2011, se celebró el Año internacional de los Afrodescendientes por la ONU. Es en ese contexto que se desarrolló la investigación que se denominó "Elementos afrodescendientes en la religiosidad popular. El caso de san Benito de Palermo en el oriente de El Salvador" (Erquicia y Herrera, 2012). Dicho trabajo aborda los numerosos elementos culturales en El Salvador, Ios cuales tienen su origen en un pasado que poco a poco se va revelando. Tal es el caso del culto a san Benito de Palermo, que se encuentra en la zona oriental del país. Esta región, desde la época colonial estuvo conformada por diversas comunidades étnicas que le dieron forma a muchas expresiones que se practican hoy en día. Esta investigación, permite mostrar que la identidad y los elementos culturales de los afrodescendientes se mantienen vigentes en la actualidad en el territorio salvadoreño.

Siempre continuando con la temática de los estudios interdisciplinarios desde la Antropología, la Historia y la oralidad, y con la temática de las identidades afrodescendientes, se realizó el proyecto "Imaginarios y discursos de la herencia afrodescendiente en San Alejo, La Unión, El Salvador" (Erquicia et al, 2013). Este explica cómo, desde el último censo nacional de población llevado a cabo en 2007, se enumeró un total de 7.441 salvadoreños identificados como "negros de raza". Dicho conteo mostró la existencia de una comunidad afrosalvadoreña que se rehúsa a desaparecer, a pesar de las décadas de negación de la que han sido objeto, como colectividad étnica, por parte del Estado salvadoreño. Si bien el proyecto de blanqueamiento mental, social y cultural se desarrolló con éxito, en muchos poblados del territorio salvadoreño, como San Alejo, la población reconoce desde un mundo subalterno la presencia de afrodescendientes, evidenciándolos en la tradición oral, la religiosidad y la mitología, las cuales sustentan el arribo y la distribución geográfica de estos en la zona y sus alrededores. Esta presencia étnica esta generalmente asociada a agentes negativos y a una simbolización originada desde la época colonial, que se ha mantenido vigente en el imaginario social no solo de San Alejo, sino de muchos poblados del territorio salvadoreño.

Extendiendo los temas de identidades e historias locales, se llevó a cabo la investigación denominada "Historias, patrimonios e identidades en el municipio de Huizúcar, La Libertad, El Salvador" (Erquicia et al, 2014). Y es que, en la actualidad, en el territorio salvadoreño existen muchas localidades que poseen un conocimiento parcial o mínimo de sus orígenes, sus historias, sus patrimonios y demás elementos que constituyen y forman parte de sus identidades. La localidad de Huizúcar, ubicada al sur de San Salvador, se ha presentado a través de su historia como uno de los lugares con población de ascendencia indígena de habla náhuat-pipil, junto con otros municipios localizados en la misma cordillera. La villa de Huizúcar también es conocida por poseer un templo católico de la época colonial, dedicado a San Miguel Arcángel, el cual es una obra arquitectónica de gran valor patrimonial. Los elementos patrimoniales de la cultura material y las manifestaciones de la cultura viva de sus habitantes son componentes esenciales en la construcción de la historia de las poblaciones de El Salvador.

\section{Reflexiones}

Es fundamental la investigación de temáticas abordadas desde las Ciencias Sociales y Humanidades, tanto del pasado como del presente, para comprender, entender y analizar a la sociedad salvadoreña en la actualidad. Es por ello que la línea de investigación en proyectos culturales es asimismo de relevancia para el desarrollo de políticas públicas enfocadas al tratamiento de las problemáticas sociales, económicas, políticas y culturales de las diversas comunidades urbanas y rurales de El Salvador.

Así también, es sustancial recalcar el papel que juega la investigación en el desarrollo curricular de las distintas carreras profesionales, integrado al proceso de enseñanzaaprendizaje que se lleva a cabo desde la labor docente. Es en este sentido, es notable la pertinencia de las investigaciones de cátedra en aprender-investigando. En consecuencia, la universidad, como institución de educación superior, no debe perder de vista la importancia de la formación de investigadores y el desarrollo continuo de investigaciones pertinentes que demandan la resolución de los problemas la sociedad salvadoreña. 
Estudios multidisciplinarios: un esbozo de la investigación cultural en la Universidad Tecnológica de El Salvador, 1999-2013. José Heriberto Erquicia-Cruz, pp. 8-15.
Revista entorno, abril 2015, número 58.

ISSN: 2218-3345.

\section{Referencias}

Barraza Ibarra, J. (2006). Historia de la Economía de la Provincia de El Salvador, desde el siglo XVI hasta nuestros días. Siglo XX. (2006) volumen 5, San Salvador: Universidad Tecnológica. ISBN 978-99923-21-39-3.

Barraza, I.J. (2001). La gesta de Anastasio Aquino: (una aproximación histórica). San Salvador, El Salvador: Universidad Tecnológica.

Campos Lara, O. (2006). "Estado, economía y redes de poder en el oriente salvadoreño, 1876-1915". Colección Histórica No. 2, San Salvador: Universidad Tecnológica de El Salvador. ISBN 99923-21-33-4.

Cañas Dinarte, C., Rivas, R. (2005). "La ausencia del otro: las personas indígenas en la legislación salvadoreña. Conceptualización e interpretación de la definición 'indígena' en El Salvador". Colección Antropológica No. 5, San Salvador: Universidad Tecnológica de El Salvador. ISBN 99923-21-28-8.

Castillo, B. (2012). "Medicina tradicional entre los indígenas de Izalco, Sonsonate, El Salvador". Colección Ciencias Sociales, San Salvador: Universidad Tecnológica de El Salvador. ISBN 978-99923-21-74-4.

CMS (Conferencia Mundial sobre Educación Superior), (2009). Recuperado de http://www.unesco.org/es/the2009-world-conference-on-higher-education/aboutthe-conference/

Colorado, J. (2008). "Etnografía de salvadoreños migrantes en Brentwood y Hempstead, Nueva York". Colección Ciencias Sociales, San Salvador: Universidad Tecnológica de El Salvador. ISBN 978-99923-21-45-4.

Erquicia, J.H. (2005). "Investigaciones arqueológicas en la zona del golfo de Fonseca, El Salvador". Colección Arqueológica No. 2, San Salvador: Universidad Tecnológica de El Salvador. ISBN 99923-21-30-X.

Erquicia, J.H. (2008) “Proyecto de Registro y Reconocimiento de sitios arqueológicos históricos de El Salvador". San Salvador: Universidad Tecnológica de El Salvador. ISBN 978-99923-21-47-8.

Erquicia, J.H. (2009) "Proyecto de registro de Registro y Reconocimiento de sitios arqueológicos históricos de El Salvador, Fase II". En Tomo I, Recopilación Investigativa, Universidad Tecnológica de El Salvador, San Salvador, El Salvador, 2009. ISBN 978-99923-21-508 (v.1)

Erquicia, J.H. (2012) "Proyecto de registro y reconocimiento de sitios arqueológicos históricos de El Salvador. Azul índigo: Los obrajes coloniales de añil, San Vicente y La
Paz. Fase III". En: Recopilación de Investigaciones 2011 (v.3). ISBN 978-99923-21-77-5

Erquicia, J.H. (2011) "Investigación formativa como eje transversal en el aprendizaje significativo de alumnos de antropología y arqueología de la Universidad Tecnológica de El Salvador". Trabajo de graduación para optar al grado de: Maestro en Docencia Universitaria. San Salvador: Universidad Tecnológica de El Salvador.

Erquicia, J.H, y M. Herrera (2007) “Investigación arqueológica de restos arquitectónicos en ciudad vieja y diagnóstico de la valoración patrimonial del caserío El Molino y comunidad Primavera con el sitio histórico Ciudad Vieja, Suchitoto, Cuscatlán, El Salvador". San Salvador: Universidad Tecnológica de El Salvador, Concultura y Academia Salvadoreña de la Historia.

Erquicia, J.H, y Herrera, M. (2011) “Aproximación etnográfica al culto popular del Hermano Macario en Izalco, Sonsonate, El Salvador". En: Recopilación de Investigaciones 2010: tomo1/ comp. Universidad Tecnológica de El Salvador. ISBN: 978-99923-21-652 (v.1).

Erquicia, J.H, y Herrera, M. (2012) "Elementos afrodescendientes en la religiosidad popular. El caso de San Benito de Palermo en el oriente de El Salvador". En: Recopilación de Investigaciones 2011 (v.3). ISBN 978-99923-21-77-5.

Erquicia, J.H.; Herrera, M.; Effenberger, W. (2013). “Imaginarios y discursos de la herencia afrodescendiente en San Alejo, La Unión, El Salvador". En: Recopilación de Investigaciones 2012, tomo II. ISBN 978-99923-980.

Erquicia, J.H., Herrera, M. y Pleitez, N. (2014). "Historias, patrimonios e identidades en el municipio de Huizúcar, La Libertad, El Salvador". Colección de Investigaciones 31, Universidad Tecnológica de El Salvador. ISBN 97899961-48-18-7.

Escamilla, M. y Fowler, W.R. (2013). “Proyecto migraciones nahua-pipiles del postclásico en la Cordillera del Bálsamo". Colección Investigaciones No. 24. San Salvador: Vicerrectoría de Investigación, Universidad Tecnológica de El Salvador.

Gallardo, R. (2005). "Identidad española reflejada en una casa colonial de la antigua villa de San Salvador" (15281545). Colección Arqueológica No. 1, San Salvador: Universidad Tecnológica de El Salvador. ISBN 9992321-25-3.

González de Arenas, M. (2004). "Los retos de la antropología en Centroamérica". Colección Antropológica No. 2, San Salvador: Universidad Tecnológica de El Salvador. ISBN 99923-21-18-0. 
Gutiérrez, A.; Mata, M.; Osegueda, C.; Sermeño, A. (2005). "Breve estudio del proceso de elaboración de alfarería de Quezaltepeque". Colección Antropológica No. 4, San Salvador: Universidad Tecnológica de El Salvador. ISBN 99923-21-24-5.

Hernández, A. (2003). "Investigación e investigación formativa". En: Revista Nómadas, Universidad Central de Colombia. Recuperado en, http://www.ucentral.edu. co/NOMADAS/nunme-ante/16-20/PdfsNomadas $\% 20$ 18/17-investigacion.PDF

LES. “Ley de Educación Superior de 1995 y Reforma en 2005. Ministerio de Educación" (Mined) (2005). Recuperado en, http://www.mined.gob.sv/

López Lago, L., Benítez, D. (2005). “Los pipiles en Metapán y su zona de influencia a través de las fuentes coloniales". Colección Histórica No. 1, San Salvador: Universidad Tecnológica de El Salvador. ISBN 99923-21-21-0.

Parra, C. (2004). "Apuntes sobre la investigación formativa". Educación y educadores, volumen 7, 2004. ISSN 01231294. Recuperado de http://dialnet.unirioja.es/servlet/ articulo? codigo $=2041050$

Ramos, E. (2000). "Breve reseña histórica de los indígenas en El Salvador". En: revista entorno. San Salvador, El Salvador: Universidad Tecnológica de El Salvador.

Ramos, E. (2001). "Breve reseña histórica de los indígenas en El Salvador: segunda parte". En: revista entorno. San Salvador, El Salvador: Universidad Tecnológica de El Salvador.
Ramos, E. (2004). "La sobrevivencia de la medicina tradicional como alternativa en los sectores populares (magia, hechicería y medicina tradicional)". En: revista entorno, agosto de 2004. San Salvador, El Salvador: Universidad Tecnológica de El Salvador, pp.30-32. ISSN: 2071-8748.

Rivas, R. (2004). "Maras y transculturación. Orígenes de la violencia entre pandillas juveniles en llobasco, El Salvador". Colección Antropológica No. 1, San Salvador: Universidad Tecnológica de El Salvador. ISBN 99923-21-17-2.

Rivas, R. (2012). "Antropología en El Salvador. Recorrido histórico y descriptivo". Colección Ciencias Sociales, San Salvador: Universidad Tecnológica de El Salvador. ISBN 978-99923-21-85-0.

Rivas, R. (2013). Ilobasco de los recuerdos. De las muchas cosas que pasaban. Colección Ciencias Sociales, San Salvador: Universidad Tecnológica de El Salvador. ISBN 978-99961-48-13-2.

Rivas, R., Gómez, E. (2005). "Antropología y arqueología de la isla Conchagüita en el golfo de Fonseca". Colección Antropológica No. 6, San Salvador: Universidad Tecnológica de El Salvador. ISBN 99923-21-27-X.

Rivas, R.; Mata, I.; Valencia, R.; Hernández, J.; Marroquín, J. (2005). "Deseo local y patrimonio histórico en Suchitoto". Colección Antropológica No. 3, San Salvador: Universidad Tecnológica de El Salvador. ISBN 99923-21-20-2. 